\title{
Comparative Study of Outcomes of Early Versus Interval Laparoscopic Cholecystectomy in Acute Calculus Cholecystitis.
}

\author{
Kirit D Parmar*, Mitul V Patel**, Sameer I Bengali** \\ *Associate Professor,Department Of General Surgery, Smt. NHL Medical College And Seth V.S. General \\ Hospital, Ahmedabad, Gujarat, India \\ **Resident Doctor,Department Of General Surgery, Smt. NHL Medical College And Seth V.S. General \\ Hospital, Ahmedabad, Gujarat, India
}

\begin{abstract}
Background And Objectives: To compare the overall efficacy, mortality and morbidity in patients managed with early versus delayed laparoscopic Cholecystectomy for acute calculus Cholecystitis.

Methods: A total of 50 patients were chosen for the study. They were selected on the bases of presence of two of the following four features: (1) Abdominal pain characteristic of Acute Cholecystitis, (2) Positive Murphy's sign, (3) Total Leucocyte Count > 10,000/ uL, and (4) Ultra-sonographic evidence of Acute Calculus Cholecystitis. Patients were selected on the bases of Initial surgical management planned for laparoscopic cholecystectomy whether in immediate or interval Cholecystectomy. Patients with other complications of Cholecystitis, other abdominal pathologies and surgeries were excluded from the study. Patients were divided into 2 study groups by odd-even method of randomization and they were categorized as Group A and Group B alternatively as per their primary surgical OPD presentation sequence. Immediate cholecystectomy was performed in Group A patients while interval cholecystectomy after initial conservative management was done in Group B patients.

Results: The mean age of presentation for acute calculus cholecystitis was found to be $48.4 \pm 14.2$ years with $36 \%$ of male patients and $64 \%$ of female patients of total patients. The Conversion of laparoscopic to open cholecystectomy rate was $8 \%$ in early cholecystectomy group as compared to $4 \%$ in interval cholecystectomy group in our study. There was a significant shorter operative duration in interval cholecystectomy as compared to early cholecystectomy. The hospital stay was significantly longer in patients of interval cholecystectomy than early cholecystectomy. The intra operative and postoperative complications like bile and gall stone spillage due to gall bladder perforation, hemorrhage, biliary fistula and wound infection were more common with early than interval laparoscopic cholecystectomy. Complications like biliary fistulas occurred in patients operated for early cholecystectomy and not in interval cholecystectomy. The requirement of antibiotics and analgesics were significantly higher in interval cholecystectomy group as compared to early cholecystectomy group. No mortalities were recorded in either group.

Interpretation and Conclusion: There is no significant difference in overall clinical outcome in patients managed by immediate and interval cholecystectomy in case of acute calculus cholecystitis. The total hospital stay and medication requirement are higher in interval cholecystectomy group as compared to early cholecystectomy group.Overall morbidity is higher in interval cholecystectomy group patients.Intraoperative difficulty, postoperative morbidity and complications are higher in immediate cholecystectomy group.Patients should be planned for immediate or interval cholecystectomy according to their symptomatic severity and willingness to undergo early surgery or initial conservative management.
\end{abstract}

Keywords: Acute calculus cholecystitis, Early, Interval laparoscopic cholecystectomy, post-operative stay, biliary fistula.

\section{Introduction}

Gall stones are one of the major causes of morbidity and mortality all over the world. Cholecystectomy is the definitive treatment for patients with acute cholecystitis. Early cholecystectomy performed within 2 to 3 days of presentation is preferred over interval or delayed cholecystectomy that is performed 6 to 10 weeks after initial medical therapy. About $20 \%$ of patients fail initial medical therapy and require surgery during the initial admission or before the end of the planned conservative period. [1]

Since last 20 years, increasing number of surgeons have favored a policy of early surgery. Several randomized studies have shown that performing early cholecystectomy for acute cholecystitis is better than delayed cholecystectomy in terms of shorter hospital stay, better operative outcome. Though recovery from disease is earlier in early cholecystectomy group, both groups have similar operative morbidity and mortality rates. [1] Cholecystectomy early on index admission is recommended over delayed elective cholecystectomy for 
most patients with acute cholecystitis, based on randomized trials and meta-analyses. Per few international studies, timing of cholecystectomy remains controversial.

The present study is an endeavor to compare the intra operative as well as post-operative morbidity and mortality and clinical outcome between early laparoscopic cholecystectomy and interval laparoscopic cholecystectomy in acute calculus cholecystitis.

\section{Material And Methods}

Acute calculus cholecystitis is one of the causes of acute abdomen. A prospective analytical study of 50 such cases during July 2014 to November 2016 was undertaken. On admission, detailed history was taken. General examination emphasized on temperature, pulse, blood pressure, jaundice, cardiovascular and respiratory system and general signs of acute cholecystitis. Abdominal signs were sought to arrive at the diagnosis.

Necessary investigations were carried out.

\section{Inclusion Criteria:}

- Patients were admitted to the study based on clinical, laboratory and radiological evidence of acute calculous cholecystitis. The diagnosis was based on the presence of two of the following four features: (1) Abdominal pain characteristic of Acute Cholecystitis, (2) Positive Murphy's sign, (3) Total Leucocyte Count $>10,000 / \mathrm{uL}$, and (4) Ultra-sonographic evidence of Acute Calculous Cholecystitis

- Admission in our unit between August, 2014 and November, 2016

- Initial surgical management planned for laparoscopic cholecystectomy whether in immediate or interval cholecystectomy

\section{Exclusion Criteria:}

- Patients with ultra-sonographic findings of common bile duct calculi/pancreatitis/gall bladder perforation/gall bladder gangrene/gall bladder abscess

- Patients with other associated abdominal pathology

- Patients with any previous abdominal surgery

- Septic shock

- Pregnancy/ Breast-feeding mothers

- Patients with any significant systemic disease

Patients were divided into 2 study groups by odd-even method of randomization and they were categorized as Group A\& Group B alternatively as per their primary surgical OPD presentation sequence. Immediate cholecystectomy was performed in Group A patients while interval cholecystectomy after initial conservative management was performed in Group B patients. In this study, the clinical findings and investigations of 2nd time admission of Group B patients were considered when they underwent surgery and not the primary data of their first symptomatic presentation.

\section{Procedure for patients receiving immediate surgery}

- Laparoscopic cholecystectomy within 72 hours after hospital admission

- Antibiotic therapy with Injectable Ceftriaxone(1g) i.v. 12 hourly and Injectable Metronidazole(500mg) i.v. 8 hourly for 48 hours post operatively followed by oral Cefixime $(200 \mathrm{mg}) \mathrm{BD}$ if the clinical condition is stable.

- Discharge of the patient as soon as possible after Day 2, if the body temperature and blood leukocyte count are normal

- Test-of-Cure visit at Day 30

Procedure for patients receiving primarily conservative therapy with elective surgery

- Antibiotic therapy with Injectable Ceftriaxone( $1 \mathrm{~g})$ i.v. 12 hourly and Injectable Metronidazole $(500 \mathrm{mg})$ i.v. 8 hourly for 24 hours followed by oral Cefixime(200mg) BD if clinical condition is stable.

- Discharge of the patient as soon as possible after starting oral cefixime

- Elective cholecystectomy between Days 35 and 70 after primary admission

- Test-of-Cure visit at Day 30

Demographics, clinical data, and findings for medical history, physical examination, laboratory tests, and ultrasonography were recorded for all patients. The patients were followed up during postoperative hospitalization. The primary evaluation criteria of the study were operative and postoperative variables, such as operation time, per-operative findings per-operative and post-operative complications, hospitalization duration (total and post-operative) and rate of conversion to open cholecystectomy. For the late operation group, 
hospitalization duration was considered as total length of stay for both first and second hospitalizations added together.

\section{Results}

table 1 age distribution

\begin{tabular}{|l|l|l|}
\hline Age Group & Number of patients & Percentage \\
\hline $21-30$ & 5 & $10 \%$ \\
\hline $31-40$ & 11 & $22 \%$ \\
\hline $41-50$ & 15 & $30 \%$ \\
\hline $51-60$ & 9 & $18 \%$ \\
\hline $61-70$ & 8 & $16 \%$ \\
\hline $71-80$ & 2 & $4 \%$ \\
\hline
\end{tabular}

The most common age of presentation is 31 to 50 years in our study group with $52 \%$ of patients belonging to this age group with mean age of $48.4 \pm 14.2$ years.Female patients comprised $64 \%$ of total cases in our study group while male patients comprised only $36 \%$ of total patients.

\section{Conversion Rate:}

2 out of 25 patients were converted to open cholecystectomy in immediate cholecystectomy group while 1 out of 25 patients were converted to open cholecystectomy in interval cholecystectomy group.

Table 2 Operative Time

\begin{tabular}{|l|l|l|}
\hline & GROUP A & GROUP B \\
\hline OPERATIVE TIME (Mins) & $87.04 \pm 11.62$ & $70.56 \pm 10.78$ \\
\hline
\end{tabular}

Mean operative time is $87.04 \pm 11.62$ minutes in early laparoscopic cholecystectomy while it is $70.56 \pm 10.78$ minutes in interval laparoscopic cholecystectomy with $\mathrm{P}$ value $<0.05$. This signifies that this variation of surgical duration is not by chance and duration of surgery is reduced in interval cholecystectomy.

\section{Recurrence of symptoms and requirement of early surgery in conservatively managed patients:}

4 out of 25 conservatively managed patients had recurrent symptoms and 2 out of those patients had to undergo surgical intervention before planned time of cholecystectomy and 1 out of them was converted to open cholecystectomy due to difficulty in laparoscopic surgery.

Table 3 Hospital Stay

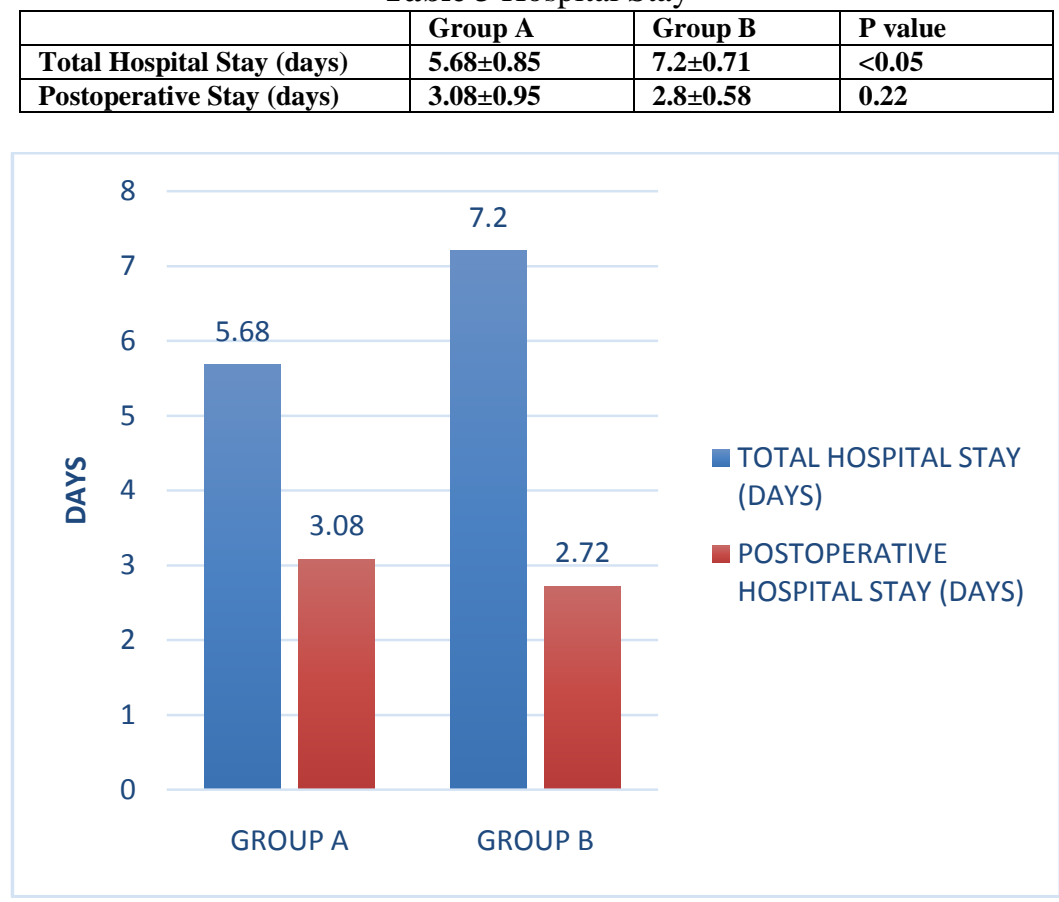

Chart 1 Hospital Stay 
Total duration of hospital stay is $5.68 \pm 0.85$ days in immediate cholecystectomy group while it is $7.2 \pm 0.71$ days in interval cholecystectomy group with $\mathrm{P}$-value $<0.05$. Postoperative hospital stay is $3.08 \pm 0.95$ days in immediate cholecystectomy group and $2.8 \pm 0.58$ in interval cholecystectomy group with $\mathrm{P}$-value $<0.05$. This suggests that total duration of hospital stay is significantly high in interval cholecystectomy group. Postoperative hospital stay is high is immediate cholecystectomy group but it is not significant as this variation could be due to chance as $p$ value of it is $>0.05$.

Table 4 Signs And Symptoms

\begin{tabular}{|l|l|l|l|}
\hline & & Group A & Group B \\
\hline \multirow{5}{*}{ Signs and Symptoms } & RHC Pain & $\mathbf{2 5}(\mathbf{1 0 0 \%})$ & $\mathbf{4}(\mathbf{1 6 \%})$ \\
\cline { 2 - 4 } & Fever & $\mathbf{1 9}(\mathbf{7 6 \%})$ & $\mathbf{2 ( 8 \% )}$ \\
\cline { 2 - 4 } & Jaundice & $\mathbf{2 ( 8 \% )}$ & $\mathbf{2 ( 8 \% )}$ \\
\cline { 2 - 4 } & Nausea/Vomiting & $\mathbf{8}(\mathbf{3 2 \%})$ & $\mathbf{0}$ \\
\cline { 2 - 4 } & RHC Tenderness & $\mathbf{9 ( 3 6 \% )}$ & $\mathbf{2 ( 8 \% )}$ \\
\cline { 2 - 4 } & Palpable GB & $\mathbf{2}(\mathbf{8 \%})$ & $\mathbf{2 ( 8 \% )}$ \\
\hline
\end{tabular}

Most of the patients had recovered of their symptoms during conservative period.

Table 5 Ultrasonographic Findings

\begin{tabular}{|l|l|l|l|}
\hline & & Group A & Group B \\
\hline \multirow{3}{*}{ USG } & Average wall thickness of GB $(\mathrm{mm})$ & $\mathbf{5 . 9 6} \pm 1.02$ & $\mathbf{4 . 5 2 \pm 0 . 5 9}$ \\
\cline { 2 - 4 } & Pericholecystic Collection & $\mathbf{3 ( 1 2 \% )}$ & $\mathbf{2 ( 8 \% )}$ \\
\cline { 2 - 4 } & Stone in Cystic Duct & $\mathbf{1 5}(60 \%)$ & $\mathbf{8 ( 3 2 \% )}$ \\
\hline
\end{tabular}

Table 6 Per-Operative Findings And Complications And Post-Operative Complications

\begin{tabular}{|l|l|l|l|}
\hline & & Group A & Group B \\
\hline \multirow{3}{*}{ Per Op Findings } & GB Adhesion & $\mathbf{1 0}(\mathbf{4 0 \%})$ & $\mathbf{3}(\mathbf{1 2 \%})$ \\
\cline { 2 - 4 } & GB Congestion & $\mathbf{6}(\mathbf{2 4 \%})$ & $\mathbf{2}(\mathbf{8 \%})$ \\
\cline { 2 - 4 } & GB Perforation & $\mathbf{2}(\mathbf{8 \%})$ & $\mathbf{0}$ \\
\hline \multirow{3}{*}{ Per Op Complication } & Bile/Stone Spillage & $\mathbf{6}(\mathbf{2 4 \%})$ & $\mathbf{2}(\mathbf{8 \%})$ \\
\cline { 2 - 4 } & Hemorrhage & $\mathbf{4}(\mathbf{1 6 \%})$ & $\mathbf{2}(\mathbf{8 \%})$ \\
\cline { 2 - 4 } & CBD Injury & $\mathbf{0}$ & $\mathbf{0}$ \\
\cline { 2 - 4 } & Conversion to Open Surgery & $\mathbf{2}(\mathbf{8 \%})$ & $\mathbf{1}(\mathbf{4 \%})$ \\
\hline \multirow{3}{*}{ Post Op Complication } & Bile Leakage & $\mathbf{2}(\mathbf{8 \%})$ & $\mathbf{0}$ \\
\cline { 2 - 4 } & Wound Infection & $\mathbf{2}(\mathbf{8 \%})$ & $\mathbf{1}(\mathbf{4 \%})$ \\
\cline { 2 - 4 } & Hemorrhage & $\mathbf{0}$ & $\mathbf{0}$ \\
\hline
\end{tabular}

Per-operative adhesion, congestion and perforation were found in more number of patients who underwent immediate cholecystectomy as well as per-operative and post-operative complications were also more in numbers in immediate cholecystectomy group.

Table 7: Requirement Of Medications During Morbidity Period

\begin{tabular}{|l|l|l|l|}
\hline & Group A & Group B & P value \\
\hline Avg. duration of inj antibiotics (Days) & $3.16 \pm 0.37$ & $3.84 \pm 0.62$ & $<0.05$ \\
\hline Avg. duration of oral antibiotics (Days) & $\mathbf{5 . 6} \pm 1.38$ & $10.6 \pm 0.82$ & $<0.05$ \\
\hline Avg. duration of inj analgesics (Days) & $\mathbf{3 . 0 8} \pm 0.28$ & $\mathbf{3 . 5 2} \pm 0.65$ & $<0.05$ \\
\hline Avg. duration of oral analgesics (Days) & 15 & $19.6 \pm 1.87$ & $<0.05$ \\
\hline
\end{tabular}

Total duration of antibiotic and analgesic medication requirements was higher in interval cholecystectomy group with $\mathrm{P}$-value $<0.05$ which is suggestive of significant higher requirements of medications.

\section{Mortality Rate}

No mortality was recorded in either of the study group in our study.

\section{Discussion}

- In this study mean age of presentation for acute calculus cholecystitis was found to be $48.4 \pm 14.2$ years with $36 \%$ of male patients and $64 \%$ of female patients of total patients.

- Conversion rate was $8 \%$ in early cholecystectomy group as compared to $4 \%$ in interval cholecystectomy group in our study. It should be noted that physical, clinical, and radiologic characteristics of patients in both study groups were similar in our study, except for body temperature and blood total leukocyte count, which were significantly higher in the early laparoscopic cholecystectomy group. A meta-analysis study 
from American journal of gastroenterology showed conversion rate to open surgery in early laparoscopic cholecystectomy 7.99\%. [2]

- In our study, we found that average operative duration was 87.04 minutes in early cholecystectomy group and 70.56 minutes in delayed cholecystectomy group with a $p$ value of $<0.05$ which is suggestive of significant shortening operative duration in interval cholecystectomy. Siddiqui et alanalyzed 4 clinical studies containing 375 patients and found shorter hospital stay and longer operation time in early laparoscopic cholecystectomy, but they found no significant difference in conversion rates between early and delayed laparoscopic cholecystectomy. [3]

- In our study $4(16 \%)$ of the patients had recurrent symptoms and $2(8 \%)$ of total 25 patients had to undergo surgical intervention before planned time of cholecystectomy with conversion rate (to open cholecystectomy) of 50\% due to difficulty in laparoscopic surgery. A study from Cochrane database revealed that $18.3 \%$ of the patients included in the delayed groups, in five RCTs had to undergo emergency surgery in the interval period for either non-resolution or recurrence of symptoms before their planned operation with $45 \%$ conversion rate to open cholecystectomy.[4] A meta-analysis study fro the American Journal of Gastroenterology showed that more than $20 \%$ of patients referred to delayed surgery failed to respond to conservative management or suffer recurrent cholecystitis in the interval period. [2]

- In our study, average total hospital stay was found to be 7.2 days in interval cholecystectomy and 5.68 days in early cholecystectomy. A meta-analysis study from American journal of gastroenterology showed that total hospital stay (mean $\pm \mathrm{SD}$ ) was significantly shorter in the early surgery group $(9.6 \pm 2.5$ days vs $17.8 \pm 5.8$ days; $\mathrm{p}<0.0001$ ). [2]In another study conducted by S.A. Khuwaith found that the average duration of hospital stay for delayed cholecystectomy to be 18.5 days. [5] One study showed that the total hospital stay was shorter by 4 days with ELC than with delayed surgery. This was due to patients in the delayed group requiring two treatment episodes, one for the conservative treatment of acute cholecystitis and another for definitive surgical treatment. In addition, many of the patients in the delayed group required emergency readmission owing to recurrent symptoms. The number of work days lost was also less with ELC in the only trial that reported this outcome. [6]

- We found that intraoperative and postoperative complications like bile and gall stone spillage due to gall bladder perforation, hemorrhage, biliary fistula and wound infection were more common with early than interval laparoscopic cholecystectomy. The higher rate of complications in the early laparoscopic cholecystectomy group may also be explained by the significantly higher initial body temperatures and blood total leukocytes count in this group. However, considering the shorter hospitalization duration and lower treatment costs, early laparoscopic cholecystectomy still seems advantageous over late intervention. In a prospective randomized study, they found more intra-operative and post-operative complication rate in early cholecystectomy group than interval cholecystectomy group similar to our study. [7]

- Out of the 25 cases of early cholecystectomy, 2 patients developed low output $(<200 \mathrm{cc})$ biliary fistula which presented as bile leak in the drain left in-situ. Both patients were managed conservatively with Inj. Hyoscine butyl bromide and frequent fatty meals. The drain output gradually decreased and patients were discharged after removal of drain when the output was negligible. The follow up of these patients was uneventful and follow up ultrasonography of abdomen did not show any collection in the gallbladder bed. None of the patients from interval cholecystectomy group had developed biliary fistula.

- In our study, overall requirement of antibiotics and analgesics were significantly higher in interval cholecystectomy group as compared to early cholecystectomy group.

- In our study, no mortality was recorded in either group.

- Patients returned to work early during post-operative period after interval cholecystectomy as compared to immediate cholecystectomy but overall morbidity is higher in patients planned for interval cholecystectomy due to recurrence of symptoms during conservative period.

\section{Conclusion}

Here, we have done prospective comparative study of clinical outcome in early and interval cholecystectomy in acute calculous cholecystitis. We have reached to following conclusion in our study.

- There is no significant difference in overall clinical outcome in patients managed by immediate and interval cholecystectomy in case of acute calculous cholecystitis.

- Total hospital stay and total medication requirement are higher in interval cholecystectomy group as compared to early cholecystectomy group.

- Overall morbidity is higher in interval cholecystectomy group patients.

- Intraoperative difficulty, postoperative morbidity and complications are higher in immediate cholecystectomy group. 
- Patients should be planned for immediate or interval cholecystectomy according to their symptomatic severity and willingness to undergo early surgery or initial conservative management.

It requires more number of cases to reach to final conclusion in this study.

\section{References}

[1]. S. Ahndt, H. Pitt, Sabiston- Textbook of Surgery, 17 edi. Cha.52, pg.1611-1612, Elsevier Saunders, 2004.

[2]. Claudio Papi MD, Marco Catarci MD, Letizia D'Ambrosio MD; Timing of Cholecystectomy for Acute Calculous Cholecystitis: A Meta-Analysis; The American Journal of Gastroenterology (2003) 99, 147-155; doi:10.1046/j.1572-0241.2003. 04002.x

[3]. Siddiqui T, MacDonald A, Chong PS, Jenkins JT. Early versus delayed laparoscopic cholecystectomy for acute cholecystitis: a meta-analysis of randomized clinical trials. Am J Surg. 2008;195(1):40-47. [PubMed]

[4]. Gurusamy KS, Davidson C, Gluud C, Davidson BR (2013) Early versus delayed laparoscopic cholecystectomy for people with acute cholecystitis. Cochrane Database Syst Rev 6:CD005440. doi: 10.1002/14651858.CD005440.pub3

[5]. Khuwaitir S.A. 1984. J.A.M. AJuly.

[6]. Lo C, Liu C, Fan ST, Lai EC, Wong J. Prospective randomized study of early versus delayed laparoscopic cholecystectomy for acute cholecystitis. Ann Surg 1998; 227: 461-467.CrossRef | PubMed |CAS | Web of Science® Times Cited: 153

[7]. Alper Bilal Özkardeş, Mehmet Tokaç, ErsinGürkanDumlu, Birkan Bozkurt, Ahmet BurakÇiftçi, FahriYetişir, and Mehmet Kılıç (2014) Early Versus Delayed Laparoscopic Cholecystectomy for Acute Cholecystitis: A Prospective, Randomized Study. IntSurg: January-February 2014, Vol. 99, No. 1, pp. 56-61. 